Original Article

\title{
Correlation among proprioception, muscle strength, and balance
}

\author{
Huinui Wang, PhD ${ }^{1)}$, Zhongqiu Ji ${ }^{1)^{*}}$, Guiping Jiang ${ }^{1)}$, Weitong LiU, MS ${ }^{1)}$, Xibian JiaO, PhD ${ }^{1)}$ \\ 1) College of Physical Education and Sports, Beijing Normal University: No.19 XinJieKouWai St.,
} HaiDian District, Beijing 100875, China

\begin{abstract}
Purpose] To study the correlation among proprioception, muscle strength, and balance. [Subjects and Methods] A balance testing system (Biodex Balance System, BBS) and an isokinetic testing system (Biodex System 4 , BS4) were used to test related indexes in 24 healthy young females. [Results] With the knee joint at 15 degree flexion, proprioception was significantly correlated with Limits of Stability-Time values, and was highly significantly correlated with Limits of Stability-Overall and Athlete Single Leg Medial/Lateral values. The sense of force was significantly correlated with Limits of Stability-Overall and Athlete Single Leg-Overall values. Quadriceps strength was significantly associated with Limits of Stability-Overall, Athlete Single Leg Medial/Lateral, and Athlete Double Leg-Overall values. The ratio of Quadriceps to Hamstring strength was significantly correlated with Athlete Single Leg Medial/Lateral, and Athlete Single Leg-Overall values. With the knee joint at $45^{\circ}$, proprioception was highly significantly correlated with dynamic balance, and was significantly correlated with double foot support under static balance; force sense had a high correlation with Limits of Stability-Overall, but no correlation with other indexes. Quadriceps strength had a significant correlation with dynamic and static balance; the ratio of Quadriceps/Hamstring had a highly significant correlation with Limits of Stability-Overall, Athlete Single LegAnterior/Posterior and Athlete Single Leg-Overall. [Conclusion] At different knee angles, the correlation differs among proprioception, force sense, quadriceps strength, the Quadriceps/Hamstring ratio, and balance. Key words: Proprioception, Muscle strength, Balance
\end{abstract}

(This article was submitted Aug. 15, 2016, and was accepted Sep. 7, 2016)

\section{INTRODUCTION}

Balance, as an essential function in human daily life and sports activities, can be defined as central nervous system input from proprioceptive, vestibular, and visual afferent nerve signals, with analysis of signal integration. Human body posture is controlled by motor neurons that regulate muscle activity ${ }^{1,2}$. Postural control can be divided into static balance and dynamic balance; static balance controls the oscillation amplitude of the body, and dynamic balance uses the body's internal and external information to analyse factors that influence the stability of interference (Such as walking, pushing and pulling), to maintain postural control. The perfect combination of static and dynamic balance is key to normal activities of the human daily life ${ }^{3)}$. Based on recent research, Fig. 1 summarizes the factors that affect balance ability ${ }^{4}$. These factors include proprioception, vestibular and visual incoming signals, central nervous system (CNS) signal analysis and comprehension ability, and muscle coordination and control ability. The factors potentially influencing postural balance include fatigue, age, gender, physical activity ability, and lower limb injury. The most commonly used methods to study balance ability in the elderly evaluate lower limb muscle strength, arthritis, and athletic knee cruciate ligament injuries. The most commonly used methods to evaluate balance ability of the knee joint include single and double foot support, swing track under conditions of dynamic or static gravity, and knee raise for proprioception ability ${ }^{5,6)}$.

*Corresponding author. Zhongqiu Ji (E-mail: jizhongqiu61@bnu.edu.cn)

(C2016 The Society of Physical Therapy Science. Published by IPEC Inc.

This is an open-access article distributed under the terms of the Creative Commons Attribution Non-Commercial No Derivatives (by-nc-nd) License $<$ http://creativecommons.org/licenses/by-nc-nd/4.0/>. 


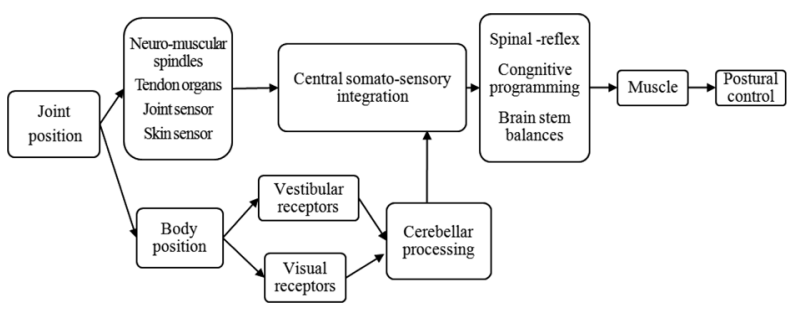

Fig. 1. Postural control system

Table 1. Mechanoreceptors for Proprioception

\begin{tabular}{llcl}
\hline Mechanoreceptors & Location & Adaptation & Function \\
\hline Pacinian corpuscles & Skin, Joint capsule, Ligaments & Quick & High frequency vibration \\
Ruffini endings & Skin, Joint capsule, Ligaments & Slow & Joint pressure \\
Unmyelinated free nerve endings & Ligaments, Related muscles & Slow & Joint pain \\
Golgi tendon organ & Joint capsule, Ligaments, Tendons, & Slow & Reflex \\
Muscle spindle & Muscle & Slow & Stretch reflex \\
\hline
\end{tabular}

Quick adaptation means that maximal impulses are observed immediately after stimulation, followed by a rapid decline. These receptors are important for sensing sudden movements, or acceleration or deceleration of a joint at the beginning or end of a motion. Slow adaptation means that a significant response or impulse is generated over a period of time with continued stimulation. These receptors function to signal change in position or actual joint position.

Anterior cruciate ligament injury in the elderly or athletes requires exercise or anterior cruciate ligament (ACL) reconstruction to improve balance ability; experimental methods proven to improve balance ability involve knee proprioception, increased knee extensor and flexor muscle strength, or improved stretch ratio in the flexor muscles ${ }^{7-10)}$. However, Robert ${ }^{8}$ tested perception of passive movement in 11 patients with ACL injury and found no significant differences among ACL injury side and the contralateral knee and a control group when postural control ability is decreased. In some patients whose motion perception level decreased, conscious increases in hamstring muscle strength compensated for the inadequacy of the position control ability after an ACL injury. Alfieri ${ }^{1)}$ showed that subjects with 12 weeks of multi-sensory training showed significant decreases in the centre of gravity of the movement area, increased power, and peak torque strength, compared with subjects who performed muscle strength training; however, the result did not explain why multi-sensory training is the most effective means of improving human balance ability.

Balance ability testing includes many components, such as static postural stability (postural stability, PS), dynamic posture stability limits (limits of stability, LOS), and dynamic fall risk (dynamic fall index, DFI). Proprioception testing methods mainly include motion perception, position perception, and force sense tests ${ }^{11,12)}$. Table 1 is a detailed explanation of the basic principles of body sensation ${ }^{9}$. Most studies only assessed the improvement of balance ability, and few articles reported on the relationship among lower limb muscle strength and various body sensory and balance tests. Therefore, this study analysed the correlation among different test methods for knee joint muscle strength, body sensation or proprioception, and posture balance.

\section{SUBJECTS AND METHODS}

Twenty-four female college students were randomly selected from Beijing Normal University, with aged $22.2 \pm 2.8$ years, height $163.8 \pm 6.0 \mathrm{~cm}$, and weight $53.3 \pm 6.9 \mathrm{~kg}$. All subjects in this experiment voluntarily, and had no significant medical conditions or knee injuries.

This study comply with the ethical standards of the Declaration of Helsinki (1975, revised 1983). Beijing Normal University Ethics Committee has approved the studies and written informed consent has been obtained from each subject.

All experiments were performed in the Beijing Normal University School of Physical Education and Sport Biomechanics Laboratory. Testing involved the knee on the dominant side. A balance testing system (Biodex Balance System, BBS; Biodex Medical Systems, USA) and an isokinetic muscle testing system (Biodex System 4, BS4) were used for measurements. The BBS system includes an angle sensor and foot position detection platform, for use in the Athlete Double Leg support stability test (ADL), Athlete Single Leg (ASL) test, and Limits of Stability (LOS) test.

BBS test: Before starting the actual test, subjects stand on the platform, to become familiar with the testing process. (1) ASL test: The test platform is set at static level, with the subject placing all weight on the right leg, placing hands naturally on the sides, and moving the right foot to screen black centre; the right leg position coinciding with the bulls eye is recorded 
and input into a computer. The subject is instructed to maintain, with eyes closed, the centre of gravity, for a test time of 20 $\mathrm{s}$, and the test is repeated 3 times $^{13}$. (2) LOS test: With the horizontal platform set at static level, the subjects stand with both feet on the platform; when the body centre of gravity and bulls eye coincide, the foot position and angle are recorded. The subjects are asked to complete the directional tasks displayed on a screen as quickly as possible, with the directions displayed at random. A total of 3 tests were performed, at intervals of $5 \mathrm{~s}$, with the third test used for results. The test results are used to determine a total score (LOS-Overall) and test completion time (LOS-Time). The higher the total score and shorter the completion time, the better the ability of the subjects to transfer the centre of gravity. (3) ADL test: Subjects stand with both feet on the platform, with the body centre of gravity and bulls eye coinciding. During the test, the subject is asked to maintain the natural standing centre of gravity without moving while keeping eyes closed. The Overall score for static stability of both feet (ADL-Overall) is recorded ${ }^{12)}$.

BS4 test: With subjects seated on the Biodex device, and the angle between the back and seat maintained at $90^{\circ}$, the right leg is attached to the power arm, and the thigh is fixed to the seat. Before starting the actual test, the subjects perform warm-up exercises. The passive isokinetic mode is set at an angular velocity of $60 \% \mathrm{~s}$, and the dynamometer drives the knee during passive warm-up; the exercise is performed 30 times, followed by the actual test after 5 minutes of rest. (1) Passive position sense test (PS): The knee joint was passively set to $15^{\circ} / 45^{\circ}$ from $90^{\circ}$ (Definition: the knee flexion position is $90^{\circ}$ and extension is $0^{\circ}$ ). The position was held for $5 \mathrm{~s}$ to allow the participants to feel the position of the knee joint in the relaxed state. The subjects moved passively to $30 / 60^{\circ}$, and pressed a button. The dynamic head moves the knee from flexion to extension. Repeated tests are carried out 3 times at each angle, at an angular velocity for passive motion of $5 \%$ s. The absolute error between the target value and the test value is taken as the test value ${ }^{14}$. (2) Force sense (FS): The torso and dominant thigh are fixed to the test chair, to remove the compensatory for movement of other body parts, with the centre of knee rotation and the dynamometer on a straight line. Test patterns for the isometric strength test are performed. The maximum voluntary isometric contraction (MVIC) was tested at $15^{\circ}$ and $45^{\circ}$ (defined as $0^{\circ}$ for the extensor). Maximum isometric flexion and extension muscle strength were tested at each knee joint angle, with test times of $5 \mathrm{~s}$, and were tested 3 times, with 5 minutes of rest between sets. The coefficient of variation (CV) requires control less than $15 \%$. After 30 minutes of rest, using $50 \%$ MVIC as the target force value, the strength of the knee joint was assessed by visual feedback of the target force on the computer screen. Subjects were then asked to close their eyes and reproduce the target Quadriceps and Hamstring force value and the measured value was recorded. The peak torque (PT) of the Quadriceps/Hamstring, the ratio of antagonistic $(\mathrm{Q} / \mathrm{H})$, and the absolute error of the difference between the target force value and the measured force were recorded. Testing was performed 2 times $^{15)}$.

SPSS 13.0 statistical software was used for analysis of the test parameters, and the parameters were analysed using Pearson's correlation coefficient. All test indexes are expressed as mean \pm standard deviation; $p<0.05$ indicates that the difference is statistically significant.

\section{RESULTS}

When the knee joint is set at $15^{\circ}$, position sense was significantly correlated with LOS-Time value $(\mathrm{p}<0.05)$, and was highly significantly correlated with LOS-Overall and ASL-ML $(\mathrm{p}<0.01)$. Force sense was significantly correlated with LOS-Overall $(\mathrm{p}<0.05)$, and was significantly associated with ASL-Overall $(\mathrm{p}<0.01)$. Quadriceps strength was significantly associated with LOS-Overall, ASL-ML, and ADL-Overall $(\mathrm{p}<0.01)$. The ratio of Quadriceps and Hamstring strength $(\mathrm{Q} / \mathrm{H})$ was significantly correlated with ASL-ML $(\mathrm{p}<0.01)$, and was significantly correlated with ASL-Overall $(\mathrm{p}<0.05)($ Table 2$)$.

When the knee joint is located at $45^{\circ}$, position sense shows a highly significant correlation with dynamic balance $(p<0.01)$, and has a definite correlation with double foot support under static balance $(\mathrm{p}<0.05)$; force sense has a high correlation with LOS-Overall, but has no correlation with any other index; Quadriceps strength has a definite correlation with dynamic and static balance $(\mathrm{p}<0.05)$; the ratio of $\mathrm{Q} / \mathrm{H}$ has a very significant correlation with LOS-Time, LOS-Overall, ASL-AP, and ASL-Overall $(\mathrm{p}<0.01)$ (Table 3).

\section{DISCUSSION}

Proprioception can be described as central nervous system input from the joints, ligaments, muscles, tendons, and skin mechanical receptors; these transmit nerve impulses, eventually causing a corresponding reflex and control of motion ${ }^{16)}$. Proprioceptive receptors can be divided into slow and fast types. Fast receptors can react quickly to external stimuli and issue nerve impulses; these include the Pacinian corpuscles, whose main function is to sense movement during the process of acceleration or deceleration motion, and the beginning or end of movement. Slow receptors continue to sense stimuli over time, and include Ruffini corpuscles, Unmyelinated nerve endings, Golgi tendon organs, and muscle spindles, whose main effect is to sense change of position and accurate positioning of joints ${ }^{17,18)}$.

At different positions of the knee joint, the extent of the role of proprioception is different ${ }^{19}$ ). At the end of the position of the knee joint, in order to prevent extreme sports injuries, all receptors will be activated; in the middle of the range of motion, the main mobilizing muscle spindle receptors sense the change in position of the knee joint ${ }^{17,20,21)}$. Therefore, this paper evaluated the relationship among knee joint proprioception, flexion strength ratio, and balance using different test methods, 
Table 2. The correlation analysis of knee joint proprioception, strength, and balance at a position of $15^{\circ}$

\begin{tabular}{lcccccc}
\hline & LOS-Time & LOS-Overall & ASL-AP & ASL-ML & ASL-Overall & ADL-Overall \\
\hline PS & $0.03^{* *}$ & $0.00^{* *}$ & 0.57 & $0.00^{* *}$ & 0.19 & 0.30 \\
FS & 0.63 & $0.01^{* *}$ & 0.08 & 0.49 & $0.01^{* *}$ & 0.33 \\
QF & 0.70 & $0.01^{* *}$ & 0.76 & $0.01^{* *}$ & 0.31 & $0.00^{* *}$ \\
Q/H & 0.93 & 0.62 & 0.85 & $0.00^{* *}$ & $0.04^{* *}$ & 0.08 \\
\hline
\end{tabular}

*Mean significant correlation $(\mathrm{p}<0.05), * *$ Mean very significant correlation $(\mathrm{p}<0.01)$ (2-tailed)

PS: position sense; FS: force sense; QF: quadriceps force; Q/H: quadriceps/hamstrings; LOS-Time: time of completed limits of stability; LOS-Overall: overall score of limits of stability; ASL-Overall: single leg of stability; ASL-ML: score of medial/lateral; ASL-AP: score anterior /posterior; ADL-Overall: overall score of double foot support

Table 3. The results of analysis of knee joint proprioception, strength, and balance at a position of $45^{\circ}$

\begin{tabular}{lcccccc}
\hline & LOS-Time & LOS-Overall & ASL-AP & ASL-ML & ASL-Overall & ADL-Overall \\
\hline PS & $0.00^{* *}$ & $0.00^{* *}$ & 0.16 & 0.58 & 0.49 & $0.04^{*}$ \\
FS & 0.44 & $0.00^{* *}$ & 0.74 & 0.14 & 0.57 & 0.66 \\
QF & $0.05^{*}$ & 0.66 & $0.00^{* *}$ & 0.41 & $0.00^{* *}$ & $0.04^{*}$ \\
Q/H & $0.05^{*}$ & $0.00^{* *}$ & $0.00^{* *}$ & 0.23 & $0.00^{* *}$ & 0.97 \\
\hline
\end{tabular}

*Mean significant correlation $(\mathrm{p}<0.05), * *$ Mean very significant correlation $(\mathrm{p}<0.01)(2$-tailed)

PS: Position sense; FS: Force sense; QF: Quadriceps force; Q/H: Quadriceps/Hamstrings; LOS-Time: Time of completed limits of stability; LOS-Overall: Overall score of limits of stability; ASL-Overall: Single leg of stability; ASLML: Score of medial/Lateral; ASL-AP: Score anterior/posterior; ADL-Overall: Overall score of double foot support

at $15^{\circ}$ and $45^{\circ}$ knee positions.

The knee plays an important role in maintaining postural balance. The Biodex System 4 tests the knee extensors (mainly quadriceps), flexors (mainly hamstrings), and peak torque at $15^{\circ}$ and $45^{\circ}$ position, and calculates the extensor and flexor ratio $(\mathrm{Q} / \mathrm{H})$. Under extensor muscle force reaction conditions, the $\mathrm{Q} / \mathrm{H}$ ratio reflects joint activities and antagonistic muscle group balance.

In the balance postural limit of stability test (LOS), the shorter the time in which the LOS-Time is performed, the faster the shift of the centre of gravity, the higher the LOS-Overall value, the better the shift of focus, and the better the control ability. When the single foot support, ASL-AP, ASL-ML, and ASL-Overall value is lower, the single foot support stability is better. In the stability test for double foot support, the lower the ADL-Overall value, the better the stability. The LOS test is representative of dynamic postural balance as ADL and ASL represents static postural balance.

When the knee joint was located at $15^{\circ}$, position sense was significantly correlated with LOS-Time value ( $\left.<<0.05\right)$, and was very significantly correlated with LOS-Overall and ASL-ML $(p<0.01)$. Force sense was significantly correlated with LOS-Overall $(\mathrm{p}<0.05)$, and was also significantly associated with ASL-Overall $(\mathrm{p}<0.01)$. Quadriceps strength was significantly associated with LOS-Overall, ASL-ML, and ADL-Overall $(\mathrm{p}<0.01)$. The ratio of Quadriceps and Hamstring strength $(\mathrm{Q} / \mathrm{H})$ was significantly correlated with ASL-ML $(\mathrm{p}<0.01)$, and was significantly correlated with ASL-Overall $(\mathrm{p}<0.05)$. This illustrates dynamic balance and proprioception and control of the direction control of foot support with very high correlation. This shows that proprioception has a significant correlation with dynamic balance and lateral direction with single foot support. When the knee joint is at $45^{\circ}$ flexion, position sense has a highly significant correlation with dynamic balance $(p<0.01)$, and has a definite correlation with double foot support under static balance $(\mathrm{p}<0.05)$; force sense has a high correlation with LOS-Overall, but has no correlation with any other index; quadriceps strength has a definite correlation with dynamic and static balance $(\mathrm{p}<0.05)$; the ratio of $\mathrm{Q} / \mathrm{H}$ has a very significant correlation with LOS-Time $(\mathrm{p}<0.05)$, LOS-Overall, ASLAP, and ASL-Overall $(\mathrm{p}<0.01)$. From the above analysis, we can conclude that at different angles, the relationship among proprioception, strength, and the ratio of $\mathrm{Q} / \mathrm{H}$ and balance is different. position sense adjusts the posture by sensing changes in the position of each part of the body, and force sense is stimulated by sensing the tension of the muscles. Therefore, when subjects are tested for their ability to feel and balance, one method alone cannot fully reflect the ability of proprioception and balance.

At different knee angles, the correlation among position sense, force sense, quadriceps strength, and Q/H ratio and balance is different.

When the knee joint was located at $15^{\circ}$, position sense was significantly correlated with LOS-Time value $(p<0.05)$, and was very significantly correlated with LOS-Overall and ASL-ML $(\mathrm{p}<0.01)$. Force sense was significantly correlated with LOS-Overall $(\mathrm{p}<0.05)$, and was significantly associated with ASL-Overall $(\mathrm{p}<0.01)$. Quadriceps strength was significantly associated with LOS-Overall, ASL-ML, and ADL-Overall $(\mathrm{p}<0.01)$. The ratio of Quadriceps and Hamstring strength $(\mathrm{Q} / \mathrm{H})$ 
was significantly correlated with ASL-ML $(\mathrm{p}<0.01)$, and was significantly correlated with ASL-Overall $(\mathrm{p}<0.05)$. When the knee joint is at $45^{\circ}$ flexion, position sense shows a highly significant correlation with dynamic balance $(p<0.01)$, and has a significant correlation with double foot support under static balance $(\mathrm{p}<0.05)$; force sense has a high correlation with LOS-Overall, but has no correlation with any other index; quadriceps strength has a significant correlation with dynamic and static balance $(\mathrm{p}<0.05)$; the ratio of $\mathrm{Q} / \mathrm{H}$ has a highly significant correlation with LOS-Time value $(\mathrm{p}<0.05)$, LOS-Overall, ASL-AP, ASL-Overall $(\mathrm{p}<0.01)$.

\section{ACKNOWLEDGEMENT}

The National Social Science Foundation for the education of general subjects, an educational intervention research for promoting and influencing the development of children's action (BLA150063).

\section{REFERENCES}

1) Alfieri FM, Riberto M, Gatz LS, et al.: Comparison of multisensory and strength training for postural control in the elderly. Clin Interv Aging, 2012, 7: 119-125. [Medline] [CrossRef]

2) Woollacott M, Shumway-Cook A: Attention and the control of posture and gait: a review of an emerging area of research. Gait Posture, 2002, 16: 1-14. [Medline] [CrossRef]

3) Lacour M, Bernard-Demanze L, Dumitrescu M: Posture control, aging, and attention resources: models and posture-analysis methods. Neurophysiol Clin, 2008, 38: 411-421. [Medline] [CrossRef]

4) Jerosch J, Prymka M: Proprioception and joint stability. Knee Surg Sports Traumatol Arthrosc, 1996, 4: 171-179. [Medline] [CrossRef]

5) Shashank MD, Ishan G: Effects of joint stabilizers on proprioception and stability - a systematic review and meta-analysis. Phys Ther Sport, 2016, 21: 64-68.

6) Fridén T, Roberts D, Ageberg E, et al.: Review of knee proprioception and the relation to extremity function after an anterior cruciate ligament rupture. J Orthop Sports Phys Ther, 2001, 31: 567-576. [Medline] [CrossRef]

7) Barrack RL, Skinner HB, Buckley SL: Proprioception in the anterior cruciate deficient knee. Am J Sports Med, 1989, 17: 1-6. [Medline] [CrossRef]

8) Beard DJ, Kyberd PJ, Fergusson CM, et al.: Proprioception after rupture of the anterior cruciate ligament. An objective indication of the need for surgery? J Bone Joint Surg Br, 1993, 75: 311-315. [Medline]

9) Xu D: The effects of Tai Chi exercise on proprioception and neuromuscular responses in the elderly people. In: Hong Y (ed.) Ann Arbor: ProQuest Dissertations Publishing, 2003.

10) Xu D, Hong Y, Li J, et al.: Effect of tai chi exercise on proprioception of ankle and knee joints in old people. Br J Sports Med, 2004, 38: 50-54. [Medline] [CrossRef]

11) Arnold BL, Schmitz RJ: Examination of balance measures produced by the biodex stability system. J Ath1 Train, 1998, 33: 323-327. [Medline]

12) Glave AP, Didier JJ, Weatherwax J, et al.: Testing postural stability: are the star excursion balance test and biodex balance system limits of stability tests consistent? Gait Posture, 2016, 43: 225-227. [Medline] [CrossRef]

13) Sibley KM, Straus SE, Inness EL, et al.: Balance assessment practices and use of standardized balance measures among Ontario physical therapists. Phys Ther, 2011, 91: 1583-1591. [Medline] [CrossRef]

14) Borsa PA, Lephart SM, Irrgang JJ, et al.: The effects of joint position and direction of joint motion on proprioceptive sensibility in anterior cruciate ligamentdeficient athletes. Am J Sports Med, 1997, 25: 336-340. [Medline] [CrossRef]

15) Dover G, Powers ME: Reliability of joint position sense and force-reproduction measures during internal and external rotation of the shoulder. J Athl Train, 2003, 38: 304-310. [Medline]

16) Todd S, Ellenbecker GJ, Jake B: Proprioception and neuromuscular control. 2012, 524-547.

17) Lee HM, Cheng CK, Liau JJ: Correlation between proprioception, muscle strength, knee laxity, and dynamic standing balance in patients with chronic anterior cruciate ligament deficiency. Knee, 2009, 16: 387-391. [Medline] [CrossRef]

18) Hewett TE, Paterno MV, Myer GD: Strategies for enhancing proprioception and neuromuscular control of the knee. Clin Orthop Relat Res, 2002 , (402): 76-94. [Medline] [CrossRef]

19) Angoules AG, Mavrogenis AF, Dimitriou R, et al.: Knee proprioception following ACL reconstruction; a prospective trial comparing hamstrings with bonepatellar tendon-bone autograft. Knee, 2011, 18: 76-82. [Medline] [CrossRef]

20) Proske U: Kinesthesia: the role of muscle receptors. Muscle Nerve, 2006, 34: 545-558. [Medline] [CrossRef]

21) Johnson KO, Popović D, Riso RR, et al.: Perspectives on the role of afferent signals in control of motor neuroprostheses. Med Eng Phys, 1995 , 17: 481-496. [Medline] [CrossRef] 